\title{
Studies on Adding MES Identification Code in AIS Information
}

\author{
LI Xian-qiang, LIU Xiao-pei and LIU Bang-sheng \\ Qingdao Ocean Shipping Mariners College, Qingdao, China
}

\begin{abstract}
This article puts forth suggestion that the ship Inmarsat identification code be added to the data structure of A and B class ship equipment of AIS for the prompt and effective communication with ships in distress and those around via Inmarsat in the process of search and rescue through MRCC (Maritime Rescue Coordination Center).
\end{abstract}

Key words: AIS, MRCC, MES ID (IMN).

\section{Introduction}

It is of great importance for the ships to be successfully rescued that the MRCC (Maritime Rescue Coordination Center) set up prompt and dependable communication with ships in distress and those around in the process of search and rescue. It is critical to acquire the identification of the relevant ships for establishing prompt and dependable communication with the ships in distress and those in the vicinity. There are at present many ways for the identification, but it has become the first choice for MRCC and ships in the vicinity to acquire the important ship information including the identification by means of the AIS (Automatic Identification System) and its relevant derived systems.

\section{The Application and Development of AIS in MRCC}

AIS is not only capable of vivid and visual display of the ship information, but also is characterized in convenient operation. Therefore, for the convenience of search and rescue of the ships in distress, the

Corresponding author: LI Xian-qiang, associate professor, dean of navigation department, research fields: teaching, studies and research in Navigation Technology; LIU Xiao-pei, professor, vice dean of navigation department, research fields: teaching, studies and management of maritime English; LIU Bang-sheng, professor, research field: teaching and studies of maritime communication.
MRCC's of many countries established the independent AIS monitor and control system and which has become the main and common means for MRCC monitor on ships and also the main means for acquiring the basic ship information.

AIS conducts information transmission via VHF and its theoretical communication distance is around 25 nautical miles. But when the antenna of the base station is in a higher position, the distance will be lengthened. With the development of satellite sounding technologies, the AIS sounding scope of MRCC has been enlarged to the whole world.

\section{The Defects of the Ship Identification Contained in AIS Information}

AIS information mainly includes the ship static and dynamic information, voyage and safety. Ship identification is a part of AIS static information, in which, ship identification includes ship name, call number, MMSI (Maritime Mobile Service Identity) and ship IMO serial number, etc., and what can be used in modern communication is MMSI. While the MMSI is only used in ground communication system, this means that when shore based administration is conducting search and rescue of the ships in distress, they can only use the equipment of the ground communication system. 


\section{The Problems in Existence for the Ground Communication System}

The ground communication is mainly composed of MF, HF and VHF communication systems and using the ground communication system brings forth the following problems.

\subsection{MF and HF Systems}

The present MMSI provided by the AIS information is mainly used in the DSC and terminals of the ground system and follow-up communication cannot be conducted on DSC terminals, which have to be transferred by the radio operator to telephone or telex channels. In the process of communication, ship-shore operators have to fix and change the working frequencies in accordance with the time, position, season, climate and distance of ship-shore distance of communication, resulting in very complicated operational procedures. In addition, what is transmitted via the NBDP terminals is text message and it is hard to express the real intention for both sides in comparison with the form of telephone communication.

The MF/HF system makes use of the simulated communication system in conducting the transmission through antenna and ground wave. Their transmission channels and characteristics result in low quality in communication. Meanwhile, on account that the channels of MF/HF frequencies are congested and the mutual interference between stations is a problem that calls for consideration in the process of practical communication.

In search and rescue, attention should be paid to the non-proficiency in operating the $\mathrm{MF} / \mathrm{HF}$ communication equipment. Although $\mathrm{MF} / \mathrm{HF}$ communication equipment is mandatory in A 3 areas, however, with the development of ship communication equipment, $\mathrm{MF} / \mathrm{HF}$ equipment is seldom used in practical communication. As a result, seafarers are unfamiliar with $\mathrm{MF} / \mathrm{HF}$ system equipment, and in turn, they fell it a difficulty in establishing communication. Especially the distress communication using NBDP, the result is hard to imagine for seafarers in non-English speaking countries.

\subsection{VHF Equipment}

In accordance with the distance calculation for ships in VHF channels provided by appendix 3 of the IMO A. 801 (19) resolution, the distance for VHF communication is generally 25 nautical miles. This is also the A 1 sea area determined by the maritime administration of most countries. The distance is quite short.

In addition, on account that AIS adopts the digital communication techniques, while VHF uses the simulated communication technique and the latter better functions than the former. Therefore, the AIS communication distance is greater than that of telephone. In the process of search and rescue, MRCC, although may detect the information of the ships surrounding those in distress or in danger in the AIS monitor system, but further contact using VHF may prove a failure.

And, VHF frequency range is also congested and is subject to interference of other stations.

\section{The Advantages of Satellite Communication Is Search and Rescue}

The satellite communication system in present use on ships mainly includes the Inmarsat system and Iridium mobile satellite communication system.

The Inmarsat system has been in use of service to shipping for more than 30 years and has provided quality service. But its disadvantage is that it cannot provide communication service for polar areas.

The Iridium satellite system is produced by the UAS Motorola Company, as the first in the world the low Earth orbit mobile communication system. The system has a low Earth orbit satellite net and is able to provide high quality speech and digital communication service covering the whole world 
(including the polar areas). Presently, the system has found its extensive use on ships and is an important component for future GMDSS.

Because satellite excels the ground communication system in quality and anti-interference and is characterized in multi means of communication, longer distance and convenience in ship-shore operation, MRCC prefers using the satellite communication when contacting ships outside the areas covered by shore based VHF stations. In addition, with the application of net technology and satellite detecting AIS techniques and the increase of the shore based detecting scope, the satellite system is capable of real time and all weather communication with ships detected, without worrying about the influence of the factors as distance, etc.

The Inmarsat satellite communication reveals its importance in safety and distress communication through the fixing of the communication levels.

In accordance with STCW Convention, ships sailing in A 3 areas are mandatorily equipped with Inmarsat-C ship stations for providing telex and low speed data transmission, while many shipping companies additionally fix the Inmarsat- $F$ and Inmarsat pilot stations in accordance with the practical need of communication.

Inmarsat-F ship station can not only provide telephone, facsimile and data transmission, but also provide better service in the aspects of distress and safety communication. On basis of achieving 4 ship-shore priority communication levels, it also achieves the priority level of shore-ship communication. MRCC has the absolute communication priority, i.e. in the process of communication, MRCC is able to stop other ship specific communication lines for direct contact with it, which improves the leading position of MRCC in the process of search and rescue.

In general, in the process of MRCC communication for search and rescue, communication with the target ship is of convenience as long as the satellite identification code is acquired. For the various ship stations, the difference lies in communication means.

\section{MRCC Problems in Using Satellite Communication}

In the process in which MRCC conducts danger verification and facilitates the ships around to participate in the rescue, when the target ship is within the scope of AIS monitor, MRCC working staff usually communicate using VHF telephone. On the failure of communication, MRCC will use other means to contact the ship. Where the target ship is outside the scope beyond AIS monitor, MRCC will directly use other means other than VHF to contact the relevant ship. In both cases, the first choice for MRCC is the satellite communication system.

But because people cannot make use of the existing AIS monitor means to directly acquire the satellite identification code of the relevant ships, MRCC usually consults the MMSI data bank or seek assistance from the agency or shipowner before establishing contact through the satellite communication system. In so doing, the consultancy will waste time for rescue and on account of the inaccuracy information incurred by the untimely updating of the MMSI data bank, it proves difficult to contact the relevant ships. The solution might be to add the satellite identification code to AIS data format.

\section{Prospects in Adding the Satellite Identification Code to the Shipboard AIS}

In the present situation, the shipboard AIS data chain is in a state of full load and there is no data bit for the additional information. But this does not mean no information transmission can be added to the existing data format. The analysis on the AIS data format will allow the transmission of satellite identification information along the AIS data chains.

\subsection{Analysis on AIS Message 5}

The information transmitted in the AIS data chain 
includes static, dynamic information, short messages of voyage and safety. Of these, the static and voyage information belong to Message 5, while the AIS information contains the ship name, call number, ship MMSI code and IMO serial numbers, etc. AIS voyage information mainly includes the destination port, ship trimming and species of dangerous cargoes, etc. Message 5 has 242 bits and takes up 2 slot times, as shown in Table 1.

By analyzing Table 1, it can be seen that, in Message 5, the two pieces of information that take up the most bits are ship name in the static information and the port of call in voyage information, respectively 120 bits for each, indicating 20 characters. Due to the difference in encoding, the MMSI code and IMO code in static information are both 9 digits, taking up 30 bits and the IMN code capable of transmitting 9 digits also takes up 30 bits. The Iridium pilot ship station identification code transmitting 12 digits only takes up 40 bits.

7.2 The Information Types in AIS Message 5 for Future Satellite Ship Station Identification Code

In the process of transmission, the static information of AIS and ship voyage belongs to Message 5, with the same amount of parameters in the chains, but different requirements for the fixation of information. The static information of AIS is usually fixed by the engineer in the process of equipment installation and cannot be modified by the seafarers on completion. The AIS voyage information is fixed in each voyage in accordance with the practical situation of the ship.

When the identification code of the satellite ship station is treated as the static information in AIS Message 5, its advantage is that the information is not to be changed, while its disadvantage is the identification code has to be manually modified by specialists when the ship renews its satellite communication equipment. If the satellite identification code is treated as the voyage information in AIS Message 5, then on the renewal of the satellite communication equipment, seafarers will find it easier to modify the IMN. But there will be cases of miss modification, which will result in negative influence on communication. Therefore, the authors suggest that, it will be feasible to add the satellite ship station code as a part of static information to AIS Message 5.

Table 1 Static information of AIS.

\begin{tabular}{|c|c|c|}
\hline Parameters & No. of bits & Remarks \\
\hline Message identification & 6 & Identifier for Message 5 \\
\hline Transmission display code & 2 & The times for the repeater to indicate the retransmission of the message, $0-3$ \\
\hline User identification & 30 & MMSI number \\
\hline AIS version indicator & 2 & $0=$ station uses version $0 ; 1-3=$ station uses AIS versions $1,2,3$ \\
\hline IMO number & 30 & 1 999999999; 0 = N/A= default; \\
\hline Call sign & 42 & 7 ×6 bit ASCII characters; “@@@@@@@@”=N/A=default \\
\hline Ship name & 120 & $\begin{array}{l}\text { Maximum } 20 \text { characters 6 bit ASCII, } \\
\text { “@@@@@@@@@@@@@@@@@@@@” = N/A= preinstalling }\end{array}$ \\
\hline Ship and cargo type & 8 & $0=$ N $/$ A or no ship $=$ default \\
\hline Position reference & 30 & Report the position reference point \\
\hline Electronic positioning equipment type & 8 & $\begin{array}{l}0=\text { undefined (preinstalling); } 1 \text { = GPS; } 2 \text { = GLONASS; } 3 \text { = integrated } \\
\text { GPS/GLONASS; } 4 \text { = loran; } 5 \text { = Decca; } 6 \text { = integrated GPS system; } 7= \\
\text { observation; 8-15 = N/A }\end{array}$ \\
\hline Estimated time of arrival & 20 & MMDDHHMM UTC \\
\hline Deepest static water consumption & 8 & Unit 0.1 meter; 255 = draft 25.5 meters or more; $0=\mathrm{N} / \mathrm{A}$ or default \\
\hline Port of call & 120 & $\begin{array}{l}\text { Maximum } 20 \text { characters 6 bit ASCII; } \\
\text { “@@@@@@@@@@@@@@@@@@@@”=N/A=default }\end{array}$ \\
\hline Data terminal equipment & 1 & Data terminal equipment ( $0=$ available, $1=\mathrm{N} / \mathrm{A}=$ default $)$ \\
\hline Spare code & 1 & Unused, fixed at 0 \\
\hline Total No. of bits & 424 & Take up 2 slot times \\
\hline
\end{tabular}




\subsection{Source of Information Bit Taken by IMN Code}

In accordance with the encoding rules of MMSI and IMO codes in AIS system, the transmission of 9 digits requires 30 bits and 60 bits is capable of transmitting 18 digits identification code. This will satisfy the transmission requirements of 9 digits IMN in AIS data chain or 12 digits of Iridium pilot ship station identification code.

But in the present AIS data chain, there is little room for further use. Considering the influence on the safety of navigation caused by modifying the data structure of AIS, the authors suggest that 60 bits be taken out from the port of call information in voyage information of AIS Message 5 for the transmission of IMN or Iridium code.

In the present AIS Message 5, 120 bits are reserved for the transmission of port of call. In accordance with the encoding method of AIS, 20 characters at most can be transmitted. When 60 bits are used for transmitting the satellite identification code, the left over 60 bits can be used for transmitting 10 characters. When writing this paper, the authors consulted the "World port directory" and found that most of the ports occupy less than 10 characters and very few exceed 10 characters. Even some ports exceed 10 characters, abbreviations can be used and which, therefore, produces no influence on navigation safety. In addition, as far as the fixation of the voyage information is concerned, out of the consideration of business secret of ship owners, few ships will really enter their port of call. As a result, to take out 60 bits from 120 bits of the port of call information for the transmission of IMN code is the best choice.

In so doing, the AIS data chain can either be used for the transmission of satellite identification code and will render no influence on the safe navigation of ships.

\section{Conclusion}

The ship monitor system based on AIS is capable of supervision the dynamics of ships and plays a critical role in the search and rescue. However, it was made in the end 80's and beginning 90's last century and people tend to choose the ground communication system. With the development of communication technologies, the communication models provided by the ground communication system are being regionalized and even eliminated. Presently, in the process of the modernization of GMDSS, the experts still hold doubts in the role that MF/HF plays in the communication of ships. There is greater call for eliminating MF/HF equipment in ship communication system. As a result, it is a choice to add IMN identification in AIS data chain.

However, the modification of AIS data chain concerns IMO, ITU and other international organizations. Therefore, it is the wish of the authors that the administration will take into consideration the role that AIS ship monitor system plays in the search and rescue and the change of the ship communication means to submit their suggestions (proposal) and modify the existing AIS data structure, so that the IMN information becomes a part of AIS data information.

\section{References}

[1] ITU. 2010. “Recommendation ITU-R M.1731-4 (04/2010) Technical Characteristics for an Automatic Identification System Using Time-Division Multiple Access in VHF Maritime Mobile Band.” 105.

[2] Liu, B.-S. 2015. GMDSS Communication Practice. Dalian Maritime University Press, 17-8. 